\title{
Analysis of a Single Server Retrial Queue with Server Vacation and Two Waiting Buffers Based on ATM Networks
}

\author{
Shan Gao $\mathbb{D}^{1}$ and Xianchao Wang $\mathbb{D}^{2}$ \\ ${ }^{1}$ School of Mathematics and Statistics, Fuyang Normal University, Fuyang 236037, China \\ ${ }^{2}$ School of Computer and Information Engineering, Fuyang Normal University, Fuyang 236037, China \\ Correspondence should be addressed to Shan Gao; sgao_09@yeah.net
}

Received 10 June 2019; Revised 23 September 2019; Accepted 28 October 2019; Published 27 November 2019

Academic Editor: Samuel N. Jator

Copyright (c) 2019 Shan Gao and Xianchao Wang. This is an open access article distributed under the Creative Commons Attribution License, which permits unrestricted use, distribution, and reproduction in any medium, provided the original work is properly cited.

\begin{abstract}
This paper presents a discrete-time Geo/G/1 retrial queue with two waiting buffers to model an ATM network, in which the server begins a single vacation in cases where the system is empty at the instant of a service completion. New arriving customer who finds the server being on vacation can decide to either enter the retrial buffer with some probability $p$ or leave the system with complementary probability $1-p$. But the new arriving customer can begin its service immediately if he finds the server idle and join the original buffer if he finds the server busy. We first carry out an extensive analysis of the model by using the supplementary variable method and the generating function approach, and give some performance measures, such as server's state probabilities and mean queue lengths in the original buffer, retrial buffer, and in the system. Secondly, we give the generating function of the sojourn time of a customer in the system and prove that Little's law still holds in our model. Sensitivity analysis and cost optimization are finally given for illustrative purposes.
\end{abstract}

\section{Introduction}

1.1. Motivation. In this work, we consider a discrete-time retrial queue based on ATM networks. In ATM networks, the basic time unit is a binary code, all information units are transferred through the network in fixed size units, called cells (also called as "customers" in queueing terminology), and each cell contains 48 bytes of user information and 5 bytes of header. Therefore, discrete-time queueing models are more realistic models which include the discrete-time nature and other basic properties of the ATM cell, and readers are referred to Bruneel and Kim [1], Takagi [2], Ramaswami and Wang [3], Walraevens et al. [4], and references therein.

In daily life, two phenomena in ATM networks often occur: one is the retrial phenomenon, which often occurs because of the repeated requests for establishing a communication channel (called as "server") between nodes and the repeated sending of unsuccessfully received cells; the other is the vacation phenomenon, because sometimes the communication channel in ATM networks needs to be closed for some time (called as "vacation") due to lack of work, which aims to utilize the idle times for different purposes. For example, computer maintenance and testing and economizing operating cost are being assigned to do a secondary job and so forth. However, the arriving cells are often affected by the server vacation in ATM networks. When the server is on vacation, some arriving cells may temporarily leave the service area and are stored in a retrial buffer (called as orbit), where they can repeat their attempt to connect with the server some time later according to a specific retrial policy, and some nonpersistent arriving cells may leave the system forever and seek services elsewhere. Therefore, in such situation, the retrial phenomenon is incurred by the server's vacation. Based on the above characteristics of the ATM networks, we mainly focus on investigating the performance measures of such a discretetime ATM network with vacation and two waiting buffers: original buffer and retrial buffer, in which the arriving cells can begin their services if they find the server idle or are 
willing to join a waiting queue in the service area (called as an original buffer) if they find the server busy. However, when the system becomes empty, the server goes on a vacation, and some arriving cells either leave the system due to the urgent messages to be transmitted or are likely to enter the orbit to seek service later.

It should be noted that one of the motivations of this paper stems from the interesting mathematical property and its distinction from other retrial queues and vacation queues. The other motivations are its applications in the ATM networks. The advantages and contributions of this work are as follows:

(i) Model. A novel ATM network retrial queue is presented. A discrete-time Geo/G/1 retrial queue with server vacation and two waiting buffers is applied in the ATM network. This model is more suitable for reflecting the characteristics of information transmission and performance analysis for ATM networks.

(ii) Methodology and Results. We adopt the supplementary variable method to have a Markovian system and use the generating function approach to obtain the performance measures of the system and the sojourn time distribution. Little's law is proved to be true in our model.

(iii) Numerical Illustrations. Sensitivity analysis and cost optimization are included in this work for illustrative purposes.

1.2. Related Works. Since the pioneering works published in 1950 s, retrial queues have been extensively studied and used to stochastically model many problems in telecommunication, computer networks, and telephone systems. A good survey of results and fundamental methods in the area of retrial queues can be found in the books, for example, Falin and Templeton [5] and Artalejo and Gomez-Corral [6]. The papers of Artalejo [7, 8] presented a classified bibliography of the work on retrial queues, respectively, in the decades 1990-1999 and 2000-2009. Similarly, GomezCorral [9] provided a bibliographical guide to the use of matrix-analytic techniques in retrial queues. Kim and Kim [10] dealt with a survey on various continuous-time retrial queueing models, in which they presented analytic results for queue length distributions, waiting time distributions, and tail asymptotics for the queue length and waiting time distributions and considered the stability analysis of retrial queueing models. Recently, motivated by a contention problem in the downlink direction of wireless base stations in cognitive radio networks, Gao et al. [11] presented a repairable $\mathrm{M} / \mathrm{G} / 1$ retrial queue with the Bernoulli schedule and a general retrial policy. By using the solution of a Riemann boundary value problem, Dimitriou [12] derived the generating function of the stationary distribution of the number of orbiting customers at service completion epochs, and he also gave the explicit expressions for the expected delay in an orbit without solving a boundary value problem. In the study of the continuous-time retrial queueing models, the Markov process is the main mathematical tool. About more works on the Markov process, readers are referred to Dai et al. [13], Shen et al. [14], and Shen et al. [15].

The study of the retrial queues was mainly focused on the continuous-time. However, it is more realistic to use the discrete-time queues to model telecommunication networks and computer systems.

Over the past decades, Geo/G/1 queues and Geo/G/1 retrial queues with a variety of vacation policies have been well developed, such as Takagi [2], Zhang and Tian [16], Gao and Wang [17], Wang [18], Wang et al. [19], Yue and Zhang [20], Luo et al. [21], and Wang [22, 23] (not exhaustive list). However, most of these vacation queues deal with the case of single waiting queues; i.e., there is only a waiting queue in the service area in the Geo/G/1 queue or an orbit waiting queue in the Geo/G/1 retrial queue. However, it is not the case in daily life. We often see that the waiting queue in the service area and the waiting queue in the orbit may exist simultaneously, for example, in ATM networks. The works on continuous-time retrial queues with two waiting buffers with different characteristics (such as finite or infinite waiting buffer, unreliable server, and constant retrial or general retrial policy) can be found in Krishna Kumar et al. [24], Do [25], Falin [26, 27], Sherman and Kharoufeh [28] and Gao et al. [11], and references therein. However, the discrete-time retrial queues with two waiting buffers have not yet received much attention. It should be remarked that an excellent and complete study on discrete-time queueing system with two waiting buffers was presented by Li and Yang [29], in which an arriving customer either joins the waiting space with probability $\alpha$ in the service area or enters the orbit with probability $\bar{\alpha}=1-\alpha$ if he finds the server busy upon arrival. Enlightened by the previous works and the applications of discrete-time retrial queue in the ATM networks, this work is devoted to present the performance analysis of a discrete-time retrial queue with server vacation and two waiting buffers. Remarkably, the models are different in Li and Yang [29] and Gao et al.' study [11]; on the one hand, we consider the case that the customers arriving during the server busy period always join the waiting buffer in the service area; i.e., these customers are willing to wait in the service area for their services but not to choose to retry after some time later. On the other hand, the customer who arrives during the server vacation period either joins a retrial buffer with probability $p$ or leaves the system for ever with probability $\bar{p}=1-p$. To the best of our knowledge, so far, no work has been done on studying the discrete-time Geo/G/1 retrial queue with server vacation, two waiting buffers, and general retrial times by using the supplementary variable method and generating the function approach.

The outline is as follows: In Section 2, the mathematical model is introduced. In Section 3, a detailed analysis of the queueing model is presented. In Section 4, the sojourn time analysis is worked out. In Section 5, sensitivity analysis and cost optimization are given. In Section 6, conclusions are provided. 


\section{Model Description}

We consider a discrete-time Geo/G/1 queue with EAS (early arrival system, see Takagi's study [2] for details), in which there are two waiting buffers in the system: one is the original buffer in the service area and the other is the retrial buffer (called as orbit), and the server can take a single vacation.

Specifically, let the time slots be numbered by $t=0$, $1,2, \ldots$ A potential external customer arrives within the interval $\left(t, t^{+}\right)$just after the beginning of slot $t$, and a potential service completion occurs within the interval $\left(t^{-}, t\right)$ just before the beginning of slot $t$. We assume that the single vacation policy is taken by the server; that is, if the system becomes empty after a service completion, the server leaves for a vacation with random length $V$. When the vacation ends, the server immediately becomes available until the system becomes empty after a service completion once again. The vacation begins at the slot $t^{+}$after a service completion with no customer staying in the system and ends at the slot $t$. If the arriving external customer finds the server busy or idle, he immediately joins the original buffer under first-come, first-served (FCFS) discipline or begins his service. Otherwise, if the arriving external customer finds the server being on vacation, he enters the orbit with probability $p$ or leaves the system forever with probability $\bar{p}=1-p$. The customers in the orbit can access the server according to the FCFS schedule when the original buffer is empty and the server is idle. Various time epochs at which events occur are shown in Figure 1.

Thereinafter, we denote $\bar{x}=1-x$ for $\forall x \in(0,1)$. The detailed model description is as follows:

(1) Independent and identically distributed (i.i.d.) interarrival times with generic random variable $A$ follow the geometric distribution $P(A=k)=$ $\lambda \bar{\lambda}^{k-1}, k \geq 1$; i.e., $\lambda$ is the probability that there is an arrival in each slot and $\bar{\lambda}$ is the probability that no customer arrives in each slot.

(2) The service times are i.i.d. random variables with generic random variable $B$, probability mass function (p.m.f.) $P(B=k)=b_{k}, k \geq 1$, probability generating function (p.g.f.) $G_{B}(z)=\sum_{k=1}^{\infty} z^{k} b_{k}$, and $j$-th factorial moments $\mu_{j}, j=1,2$. The server can handle only one customer at a time according to the FCFS discipline.

(3) The vacation time $V$ follows general distribution with p.m.f. $P(V=k)=v_{k}, k \geq 1$, p.g.f. $G_{V}(z)=$ $\sum_{k=1}^{\infty} z^{k} v_{k}$, and $j$-th factorial moments $v_{j}, j=1,2$.

(4) Only the retrial customer at the head of the orbit can access to the server if he is idle and the original queue is empty. Successive interretrial times are i.i.d. random variables with generic random variable $R$; p.m.f. $P(R=k)=r_{k}, k \geq 0$, and p.g.f. $G_{R}(z)=$ $\sum_{k=0}^{\infty} z^{k} r_{k}$.

(5) Interarrival times, service times, vacation times, and retrial times are mutually independent.

Remark 1 (special case). When the vacation time $V$ takes fixed value 1 , the model is changed to the standard discretetime $\mathrm{Geo} / \mathrm{G} / 1$ queue.

\section{Analysis of the System}

For the discrete-time retrial queue considered in this paper, we are chiefly concerned with some performance measures: server's state probabilities, numbers of customers in the original buffer and retrial buffer, busy cycle, and sojourn time. However, our discrete-time retrial queueing model is not a Markovian queue. Thus, in this section, we introduce some supplementary variables (including the remaining retrial time, the remaining service time, and the remaining vacation time) to make the queueing model Markovian and set up a set of Kolmogorov equations satisfied by the stationary distribution of the system. Based on this, we obtain these important performance measures.

At time $t^{+}$, the state of the system can be described by the Markovian chain $X(t)=\left\{\left(N_{1}(t), N_{2}(t), U(t), J(t)\right)\right\}$, $(t \geq 0)$, where $J(t)$ denotes the state of the server, where

$$
J(t)= \begin{cases}0, & \text { the server is idle at time } t^{+}, \\ 1, & \text { the server is busy at time } t^{+}, \\ 2, & \text { the server is on vacation at time } t^{+} .\end{cases}
$$

$N_{1}(t)$ denotes the number of customers in the original queue (excluding the one in service); $N_{2}(t)$ represents the number of customers in the orbit; $U(t)$ is a supplementary variable, which denotes the remaining retrial time when $J(t)=0$ and $N_{2}(t)>0$, or the remaining service time when $J(t)=1$ or the remaining vacation time when $J(t)=2$.

The state space of the Markov chain $\{X(t), t \geq 0\}$ is $\{(0,0)\} \cup\left\{\left(n_{2}, u, 0\right), n_{2} \geq 1, u \geq 1\right\} \cup\left\{\left(n_{1}, n_{2}, u, 1\right), n_{1}, n_{2} \geq 0, u\right.$ $! \geq 1\} \cup\left\{\left(n_{2}, u, 2\right), n_{2} \geq 0, u \geq 1\right\}$, where the state $(0,0)$ denotes that the server is idle and the number of customers in the orbit is zero; the state $\left(n_{2}, u, 0\right), n_{2} \geq 1, u \geq 1$, indicates that the server is idle, $n_{2}$ customers are in the orbit, and the remaining retrial time is $u$; the state $\left(n_{1}, n_{2}, u, 1\right), n_{1}, n_{2} \geq$ $0, u \geq 1$, represents that the server is busy, there are $n_{1}$ customers in the original buffer and $n_{2}$ customers in the retrial buffer, and the remaining service time is $u$; the state $\left(n_{2}, u, 2\right), n_{2} \geq 0, u \geq 1$ denotes that the server is on vacation, $n_{2}$ customers are in the orbit, and the remaining vacation time is $u$.

Our first goal is to find the stationary distribution of the Markov chain $\{X(t), t \geq 0\}$ under the stability condition $\rho=\lambda \mu_{1}<1$ :

$$
\begin{gathered}
\pi_{0,0}=\lim _{t \longrightarrow \infty} P\left(N_{2}(t)=0, J(t)=0\right), \\
\pi_{n_{2}, u, 0}=\lim _{t \longrightarrow \infty} P\left(N_{2}(t)=n_{2}, U(t)=u, J(t)=0\right), \\
\quad n_{2} \geq 1, u \geq 1, \\
\pi_{n_{1}, n_{2}, u, 1}=\lim _{t \longrightarrow \infty} P\left(N_{1}(t)=n_{1}, N_{2}(t)=n_{2}, U(t)=u, J(t)=1\right), \\
\quad n_{1} \geq 0, n_{2} \geq 0, u \geq 1, \\
\pi_{n_{2}, u, 2}=\lim _{t \longrightarrow \infty} P\left(N_{2}(t)=n_{2}, U(t)=u, J(t)=2\right), \\
n_{2} \geq 0, u \geq 1 .
\end{gathered}
$$

For the stationary distribution of the system, the Kolmogorov equations are as follows: 


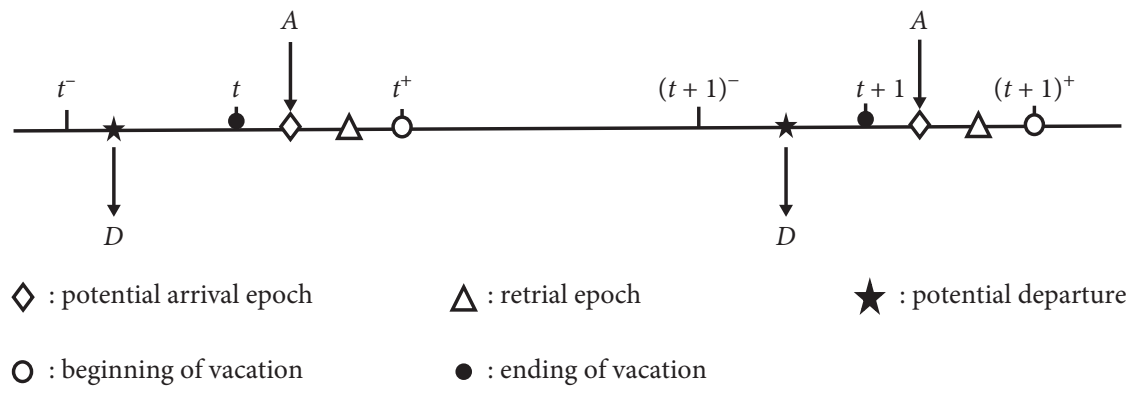

Figure 1: Various time epochs in an early arrival system (EAS).

$$
\begin{aligned}
& \pi_{0,0}= \bar{\lambda} \pi_{0,0}+\bar{\lambda} \pi_{0,1,2}, \\
& \pi_{n_{2}, u, 0}= \bar{\lambda}\left(\pi_{n_{2}, u+1,0}+r_{u} \pi_{0, n_{2}, 1,1}+r_{u} \pi_{n_{2}, 1,2}\right), \\
& n_{2} \geq 1, u \geq 1, \\
& \pi_{0, n_{2}, u, 1}= \delta_{n_{2}, 0} \lambda b_{u} \pi_{0,0}+b_{u}\left(\bar{\lambda} \pi_{n_{2}+1,1,0}+\lambda \sum_{i=1}^{\infty} \pi_{n_{2}, i, 0}\right) \\
&+b_{u}\left(\lambda \pi_{0, n_{2}, 1,1}+\bar{\lambda} \pi_{1, n_{2}, 1,1}+\bar{\lambda} r_{0} \pi_{0, n_{2}+1,1,1}\right) \\
&+\bar{\lambda} \pi_{0, n_{2}, u+1,1}+b_{u}\left(\lambda \pi_{n_{2}, 1,2}+\bar{\lambda} r_{0} \pi_{n_{2}+1,1,2}\right), \\
& n_{2} \geq 0, u \geq \\
& \pi_{n_{1}, n_{2}, u, 1}= \bar{\lambda} \pi_{n_{1}, n_{2}, u+1,1}+\lambda \pi_{n_{1}-1, n_{2}, u+1,1} \\
&+b_{u}\left(\bar{\lambda} \pi_{n_{1}+1, n_{2}, 1,1}+\lambda \pi_{n_{1}, n_{2}, 1,1}\right), \\
& n_{1} \geq 1, n_{2} \geq 0, u \geq 1, \\
& \pi_{n_{2}, u, 2}= \delta_{n_{2}, 0} \bar{\lambda} v_{u} \pi_{0,0,1,1}+\overline{\lambda p} \pi_{n_{2}, u+1,2} \\
&+\left(1-\delta_{n_{2}, 0}\right) \lambda p \pi_{n_{2}-1, u+1,2}, \quad n_{2} \geq 0, u \geq 1,
\end{aligned}
$$$$
n_{2} \geq 0, u \geq 1 \text {, }
$$

where $\delta_{n_{2}, 0}=0$ if $n_{2} \geq 1$ or 1 if $n_{2}=0$.

To obtain the performance measures of the system and analyze the sojourn time of an arbitrary customer, we define the following transforms:

$$
\begin{aligned}
\Pi_{u, 0}\left(z_{2}\right) & =\sum_{n_{2}=1}^{\infty} z_{2}^{n_{2}} \pi_{n_{2}, u, 0}, \\
\Pi_{0}\left(x, z_{2}\right) & =\sum_{u=1}^{\infty} x^{u} \Pi_{u, 0}\left(z_{2}\right), \\
\Pi_{u, 1}\left(z_{1}, z_{2}\right) & =\sum_{n_{1}=0}^{\infty} \sum_{n_{2}=0}^{\infty} z_{1}^{n_{1}} z_{2}^{n_{2}} \pi_{n_{1}, n_{2}, u, 1}, \\
\Pi_{1}\left(x, z_{1}, z_{2}\right) & =\sum_{u=1}^{\infty} x^{u} \Pi_{u, 1}\left(z_{1}, z_{2}\right), \\
\Pi_{u, 2}\left(z_{2}\right) & =\sum_{n_{2}=0}^{\infty} z_{2}^{n_{2}} \pi_{n_{2}, u, 2}, \\
\Pi_{2}\left(x, z_{2}\right) & =\sum_{u=1}^{\infty} x^{u} \Pi_{u, 2}\left(z_{2}\right) .
\end{aligned}
$$

Based on (3)-(7), we will derive the expressions of $\Pi_{0}\left(x, z_{2}\right), \Pi_{1}\left(x, z_{1}, z_{2}\right)$, and $\Pi_{2}\left(x, z_{2}\right)$ from simple to complex.

Firstly, multiplying (4) by $x^{u}$ and $z_{2}^{n_{2}}$ and summing over $u$ and $n_{2}$, we obtain

$$
\begin{aligned}
\frac{x-\bar{\lambda}}{x} \Pi_{0}\left(x, z_{2}\right)= & \bar{\lambda}\left(G_{R}(x)-r_{0}\right)\left(\Pi_{1,2}\left(z_{2}\right)+\Pi_{1,1}\left(0, z_{2}\right)\right. \\
& \left.-\left(\pi_{0,0,1,1}+\pi_{0,1,2}\right)\right)-\bar{\lambda} \Pi_{1,0}\left(z_{2}\right) .
\end{aligned}
$$

Taking $x=\bar{\lambda}$ yields that

$$
\begin{gathered}
\left(G_{R}(\bar{\lambda})-r_{0}\right)\left(\Pi_{1,2}\left(z_{2}\right)+\Pi_{1,1}\left(0, z_{2}\right)\right. \\
\left.-\left(\pi_{0,0,1,1}+\pi_{0,1,2}\right)\right)=\Pi_{1,0}\left(z_{2}\right) .
\end{gathered}
$$

Combining (9) and (10), we have

$$
\Pi_{0}\left(x, z_{2}\right)=\frac{x}{x-\bar{\lambda}} \frac{G_{R}(x)-G_{R}(\bar{\lambda})}{G_{R}(\bar{\lambda})-r_{0}} \bar{\lambda} \Pi_{1,0}\left(z_{2}\right)
$$

Secondly, it follows from (7) that

$$
\begin{aligned}
\frac{x-\left(\overline{\lambda p}+\lambda p z_{2}\right)}{x} \Pi_{2}\left(x, z_{2}\right)= & \bar{\lambda} G_{V}(x) \pi_{0,0,1,1} \\
& -\left(\overline{\lambda p}+\lambda p z_{2}\right) \Pi_{1,2}\left(z_{2}\right) .
\end{aligned}
$$

Letting $x=\overline{\lambda p}+\lambda p z_{2}$ in (12) leads to

$$
\Pi_{1,2}\left(z_{2}\right)=\frac{G_{V}\left(\overline{\lambda p}+\lambda p z_{2}\right)}{\overline{\lambda p}+\lambda p z_{2}} \bar{\lambda} \pi_{0,0,1,1} .
$$

Inserting (13) into (12), we have that

$$
\Pi_{2}\left(x, z_{2}\right)=\frac{G_{V}(x)-G_{V}\left(\overline{\lambda p}+\lambda p z_{2}\right)}{x-\left(\overline{\lambda p}+\lambda p z_{2}\right)} x \bar{\lambda} \pi_{0,0,1,1} .
$$

Thirdly, from (5) and (6), we have 


$$
\begin{aligned}
\frac{x-\left(\bar{\lambda}+\lambda z_{1}\right)}{x} \Pi_{1}\left(x, z_{1}, z_{2}\right)= & \frac{1}{z_{1} z_{2}}\left(G _ { B } ( x ) \left(z _ { 1 } \left(\lambda z _ { 2 } \left(\pi_{0,0}\right.\right.\right.\right. \\
& \left.+\Pi_{1,2}\left(z_{2}\right)+\Pi_{0}\left(1, z_{2}\right)\right) \\
& +\bar{\lambda} \Pi_{1,0}\left(z_{2}\right)+\bar{\lambda} r_{0}\left(\Pi_{1,1}\left(0, z_{2}\right)\right. \\
& \left.\left.+\Pi_{1,2}\left(z_{2}\right)-\left(\pi_{0,0,1,1}+\pi_{0,1,2}\right)\right)\right) \\
& \left.-\bar{\lambda} z_{2} \Pi_{1,1}\left(0, z_{2}\right)\right)-z_{2}\left(\bar{\lambda}+\lambda z_{1}\right) \\
& \left.\cdot\left(z_{1}-G_{B}(x)\right) \Pi_{1,1}\left(z_{1}, z_{2}\right)\right) .
\end{aligned}
$$

Putting $x=\bar{\lambda}+\lambda z_{1}$ in (15) leads to

$$
\begin{aligned}
z_{2}(\bar{\lambda} & \left.+\lambda z_{1}\right) \frac{z_{1}-G_{B}\left(\bar{\lambda}+\lambda z_{1}\right)}{G_{B}\left(\bar{\lambda}+\lambda z_{1}\right)} \Pi_{1,1}\left(z_{1}, z_{2}\right) \\
= & z_{1}\left(\lambda z_{2}\left(\pi_{0,0}+\Pi_{1,2}\left(z_{2}\right)+\Pi_{0}\left(1, z_{2}\right)\right)\right. \\
& +\bar{\lambda} \Pi_{1,0}\left(z_{2}\right)+\bar{\lambda} r_{0}\left(\Pi_{1,1}\left(0, z_{2}\right)+\Pi_{1,2}\left(z_{2}\right)\right. \\
& \left.\left.-\left(\pi_{0,0,1,1}+\pi_{0,1,2}\right)\right)\right)-\bar{\lambda} z_{2} \Pi_{1,1}\left(0, z_{2}\right) .
\end{aligned}
$$

Following from (15) and (16), we obtain that

$$
\begin{aligned}
\frac{x-\left(\bar{\lambda}+\lambda z_{1}\right)}{x} \Pi_{1}\left(x, z_{1}, z_{2}\right)= & \frac{G_{B}(x)-G_{B}\left(\bar{\lambda}+\lambda z_{1}\right)}{G_{B}\left(\bar{\lambda}+\lambda z_{1}\right)} \\
& \cdot\left(\bar{\lambda}+\lambda z_{1}\right) \Pi_{1,1}\left(z_{1}, z_{2}\right) .
\end{aligned}
$$

Now, we derive the expressions of $\Pi_{1,0}\left(z_{2}\right)$ and $\Pi_{1,1}\left(z_{1}, z_{2}\right)$, respectively, from (11) and (17). Letting $z_{1}=1$ in (16), we can get

$$
\begin{aligned}
\bar{\lambda} z_{2} \Pi_{1,1}\left(0, z_{2}\right)= & \lambda z_{2}\left(\pi_{0,0}+\Pi_{1,2}\left(z_{2}\right)+\Pi_{0}\left(1, z_{2}\right)\right) \\
& +\bar{\lambda} \Pi_{1,0}\left(z_{2}\right)+\bar{\lambda} r_{0}\left(\Pi_{1,1}\left(0, z_{2}\right)\right. \\
& \left.+\Pi_{1,2}\left(z_{2}\right)-\left(\pi_{0,0,1,1}+\pi_{0,1,2}\right)\right) .
\end{aligned}
$$

Combining (16), (17), and (18) yields that

$$
\begin{aligned}
\frac{x-\left(\bar{\lambda}+\lambda z_{1}\right)}{x} \Pi_{1}\left(x, z_{1}, z_{2}\right) \\
=\frac{\left(G_{B}(x)-G_{B}\left(\bar{\lambda}+\lambda z_{1}\right)\right)\left(z_{1}-1\right)}{z_{1}-G_{B}\left(\bar{\lambda}+\lambda z_{1}\right)} \bar{\lambda} \Pi_{1,1}\left(0, z_{2}\right) .
\end{aligned}
$$

Taking $x=1$ in (11) leads to

$$
\Pi_{0}\left(1, z_{2}\right)=\frac{\bar{\lambda}\left(1-G_{R}(\bar{\lambda})\right)}{\lambda\left(G_{R}(\bar{\lambda})-r_{0}\right)} \Pi_{1,0}\left(z_{2}\right) .
$$

Moreover, combining (3), (10), (13), (18), and (20) leads to

$$
\begin{aligned}
& \Pi_{1,1}\left(0, z_{2}\right)+\Pi_{1,2}\left(z_{2}\right)-\left(\pi_{0,0,1,1}+\pi_{0,1,2}\right) \\
& =\frac{z_{2}}{\bar{\lambda} G_{R}(\bar{\lambda})\left(1-z_{2}\right)}\left(\bar{\lambda} \pi_{0,0,1,1}-\Pi_{1,2}\left(z_{2}\right)\right) \\
& =\frac{z_{2}}{G_{R}(\bar{\lambda})\left(1-z_{2}\right)}\left(1-\frac{G_{V}\left(\overline{\lambda p}+\lambda p z_{2}\right)}{\overline{\lambda p}+\lambda p z_{2}}\right) \pi_{0,0,1,1} .
\end{aligned}
$$

Then, from (10), (11), and (21), one can have that

$$
\begin{aligned}
\Pi_{0}\left(x, z_{2}\right)= & \frac{x}{x-\bar{\lambda}} \frac{z_{2}\left(G_{R}(x)-G_{R}(\bar{\lambda})\right)}{G_{R}(\bar{\lambda})\left(1-z_{2}\right)} \\
& \cdot\left(1-\frac{G_{V}\left(\overline{\lambda p}+\lambda p z_{2}\right)}{\overline{\lambda p}+\lambda p z_{2}}\right) \bar{\lambda} \pi_{0,0,1,1} .
\end{aligned}
$$

Letting $z_{2}=0$ in (13) leads to

$$
\pi_{0,1,2}=\Pi_{1,2}(0)=\frac{G_{V}(\overline{\lambda p})}{\overline{\lambda p}} \bar{\lambda} \pi_{0,0,1,1} .
$$

Substituting (13) and (23) into (21), we can obtain

$$
\begin{aligned}
\Pi_{1,1}\left(0, z_{2}\right)= & {\left[\lambda+\bar{\lambda} \frac{G_{V}(\overline{\lambda p})}{\overline{\lambda p}}+\left(1-\frac{G_{V}\left(\overline{\lambda p}+\lambda p z_{2}\right)}{\overline{\lambda p}+\lambda p z_{2}}\right)\right.} \\
& \left.\cdot \frac{z_{2}+\bar{\lambda} G_{R}(\bar{\lambda})\left(1-z_{2}\right)}{G_{R}(\bar{\lambda})\left(1-z_{2}\right)}\right] \pi_{0,0,1,1} .
\end{aligned}
$$

Inserting (24) into (19) yields that

$$
\begin{aligned}
\Pi_{1}\left(x, z_{1}, z_{2}\right)= & \frac{x}{x-\left(\bar{\lambda}+\lambda z_{1}\right)} \\
& \cdot \frac{\left(G_{B}(x)-G_{B}\left(\bar{\lambda}+\lambda z_{1}\right)\right)\left(z_{1}-1\right)}{z_{1}-G_{B}\left(\bar{\lambda}+\lambda z_{1}\right)} \\
& \times\left[\lambda+\bar{\lambda} \frac{G_{V}(\overline{\lambda p})}{\overline{\lambda p}}+\left(1-\frac{G_{V}\left(\overline{\lambda p}+\lambda p z_{2}\right)}{\left.\overline{\lambda p}+\lambda p z_{2}\right)}\right.\right. \\
& \left.\cdot \frac{z_{2}+\bar{\lambda} G_{R}(\bar{\lambda})\left(1-z_{2}\right)}{G_{R}(\bar{\lambda})\left(1-z_{2}\right)}\right] \bar{\lambda} \pi_{0,0,1,1} .
\end{aligned}
$$

From (14), (22), and (25), we should find the probability $\pi_{0,0,1,1}$ to obtain the expressions of $\Pi_{0}\left(x, z_{2}\right), \Pi_{1}\left(x, z_{1}, z_{2}\right)$, and $\Pi_{2}\left(x, z_{2}\right)$. From (3) and (23), we know that

$$
\pi_{0,0}=\frac{\bar{\lambda}}{\lambda} \pi_{0,1,2}=\frac{(\bar{\lambda})^{2}}{\lambda} \frac{G_{V}(\overline{\lambda p})}{\overline{\lambda p}} \pi_{0,0,1,1} .
$$


Using the normalization equation $\pi_{0,0}+\Pi_{0}(1,1)+$ $\Pi_{1}(1,1,1)+\Pi_{2}(1,1)=1$, we obtain that

$$
\pi_{0,0,1,1}=K
$$

where $K^{-1}=\bar{\lambda}\left[(\bar{\lambda} / \lambda)\left(G_{V}(\overline{\lambda p}) / \overline{\lambda p}\right)+\left(\left(1-G_{R}(\bar{\lambda})\right) / G_{R}(\bar{\lambda})\right) p\right.$ $\left(\nu_{1}-1\right)+(\rho /(1-\rho))\left(1+(\bar{\lambda} / \lambda)\left(G_{V}(\overline{\lambda p}) / \overline{\lambda p}\right)+p\left(\nu_{1}-1\right) / G_{R}\right.$ $\left.(\bar{\lambda})+\nu_{1}\right]$.

Summing the above results, we have the following Theorem 1.

Theorem 1. If $\rho<1$, the joint distribution of the number of customers in the original buffer, the number of customers in orbit, the remaining retrial time, the remaining service time, and the remaining vacation time and the server state, is determined by its transform as follows:

$$
\begin{aligned}
& \pi_{0,0}=\frac{(\bar{\lambda})^{2}}{\lambda} \frac{G_{V}(\overline{\lambda p})}{\overline{\lambda p}} K \\
& \Pi_{0}\left(x, z_{2}\right)=\frac{x}{x-\bar{\lambda}} \frac{z_{2}\left(G_{R}(x)-G_{R}(\bar{\lambda})\right)}{G_{R}(\bar{\lambda})\left(1-z_{2}\right)} \\
& \left(1-\frac{G_{V}\left(\overline{\lambda p}+\lambda p z_{2}\right)}{\overline{\lambda p}+\lambda p z_{2}}\right) \bar{\lambda} K \\
& \Pi_{1}\left(x, z_{1}, z_{2}\right)=\frac{x}{x-\left(\bar{\lambda}+\lambda z_{1}\right)} \\
& \frac{\left(G_{B}(x)-G_{B}\left(\bar{\lambda}+\lambda z_{1}\right)\right)\left(z_{1}-1\right)}{z_{1}-B\left(\bar{\lambda}+\lambda z_{1}\right)} \\
& \times\left[\lambda+\bar{\lambda} \frac{G_{V}(\overline{\lambda p})}{\overline{\lambda p}}+\left(1-\frac{G_{V}\left(\overline{\lambda p}+\lambda p z_{2}\right)}{\overline{\lambda p}+\lambda p z_{2}}\right)\right. \\
& \left.\frac{z_{2}+\bar{\lambda} G_{R}(\bar{\lambda})\left(1-z_{2}\right)}{G_{R}(\bar{\lambda})\left(1-z_{2}\right)}\right] \bar{\lambda} K \\
& \Pi_{2}\left(x, z_{2}\right)=\frac{G_{V}(x)-G_{V}\left(\overline{\lambda p}+\lambda p z_{2}\right)}{x-\left(\overline{\lambda p}+\lambda p z_{2}\right)} x \bar{\lambda} K
\end{aligned}
$$

Letting $x=1$ in Theorem 1 , we have the following corollary.
Corollary 1. The expressions for the partial generating functions when the server is idle with nonempty orbit, busy, and on vacation, respectively, are as follows:

$$
\begin{aligned}
\Pi_{0}\left(1, z_{2}\right)= & \frac{z_{2}}{1-z_{2}}\left(1-\frac{G_{V}\left(\overline{\lambda p}+\lambda p z_{2}\right)}{\overline{\lambda p}+\lambda p z_{2}}\right) \\
& \cdot \frac{\left(1-G_{R}(\bar{\lambda})\right) \bar{\lambda} K}{G_{R}(\bar{\lambda})} \frac{\bar{\lambda}}{\lambda}, \\
\Pi_{1}\left(1, z_{1}, z_{2}\right)= & \frac{1-G_{B}\left(\bar{\lambda}+\lambda z_{1}\right)}{G_{B}\left(\bar{\lambda}+\lambda z_{1}\right)-z_{1}} \\
& \cdot\left[\lambda+\bar{\lambda} \frac{G_{V}(\overline{\lambda p})}{\overline{\lambda p}}+\left(1-\frac{G_{V}\left(\overline{\lambda p}+\lambda p z_{2}\right)}{\overline{\lambda p}+\lambda p z_{2}}\right)\right. \\
& \left.\cdot \frac{z_{2}+\bar{\lambda} G_{R}(\bar{\lambda})\left(1-z_{2}\right)}{G_{R}(\bar{\lambda})\left(1-z_{2}\right)}\right] \frac{\bar{\lambda} K}{\lambda}, \\
\Pi_{2}\left(1, z_{2}\right)= & \frac{1-G_{V}\left(\overline{\lambda p}+\lambda p z_{2}\right)}{\lambda p\left(1-z_{2}\right)} K .
\end{aligned}
$$

In the following subsections, we give some performance measures of our queueing system.

3.1. Steady-State Probabilities of the Server. The steady-state probabilities that the server is idle, busy, and on vacation, respectively, denoted by $P_{I}, P_{B}$, and $P_{V}$, are given by

$$
\begin{aligned}
& P_{I}=\pi_{0,0}+\Pi_{0}(1,1)=\left[\frac{\bar{\lambda}}{\lambda} \frac{G_{V}(\overline{\lambda p})}{\overline{\lambda p}}+\frac{1-G_{R}(\bar{\lambda})}{G_{R}(\bar{\lambda})} p\left(v_{1}-1\right)\right] \bar{\lambda} K, \\
& P_{B}=\Pi_{1}(1,1,1)=\frac{\mu_{1}}{1-\rho}\left(\lambda+\bar{\lambda} \frac{G_{V}(\overline{\lambda p})}{\overline{\lambda p}}+\frac{\lambda p}{G_{R}(\bar{\lambda})}\left(v_{1}-1\right)\right) \bar{\lambda} K, \\
& P_{V}=\Pi_{2}(1,1)=v_{1} \bar{\lambda} K .
\end{aligned}
$$

3.2. Mean Number Customers in Original Buffer and in Orbit. Let $L_{1,1}$ be the mean number customers in the original buffer when the server is in state 1 and $L_{2, j}$ be the mean number customers in orbit when the server is in state $j, j=0,1,2$; then, from Corollary 1, we have that 


$$
\begin{aligned}
& L_{1,1}=\left.\frac{\partial}{\partial z_{1}} \Pi_{1}\left(1, z_{1}, z_{2}\right)\right|_{z_{1}=1, z_{2}=1}=\frac{\lambda \mu_{2}}{2(1-\rho)^{2}} \\
& \cdot\left(\lambda+\frac{\bar{\lambda} G_{V}(\overline{\lambda p})}{\overline{\lambda p}}+\frac{\lambda p}{G_{R}(\bar{\lambda})}\left(\nu_{1}-1\right)\right) \bar{\lambda} K, \\
& L_{2,0}=\left.\frac{d}{d z_{2}} \Pi_{0}\left(1, z_{2}\right)\right|_{z_{2}=1}=p\left(\overline{\lambda p}\left(v_{1}-1\right)+\frac{\lambda p}{2} v_{2}\right) \\
& \frac{\left(1-G_{R}(\bar{\lambda})\right)}{G_{R}(\bar{\lambda})} \bar{\lambda} K \\
& L_{2,1}=\left.\frac{\partial}{\partial z_{2}} \Pi_{1}\left(1, z_{1}, z_{2}\right)\right|_{z_{1}=1, z_{2}=1}=\frac{\rho}{1-\rho} \frac{p}{G_{R}(\bar{\lambda})} \\
& \cdot\left(\left(\overline{\lambda p}-\bar{\lambda} G_{R}(\bar{\lambda})\right)\left(\nu_{1}-1\right)+\frac{\lambda p}{2} \nu_{2}\right) \bar{\lambda} K, \\
& L_{2,2}=\left.\frac{d}{d z_{2}} \Pi_{2}\left(1, z_{2}\right)\right|_{z_{2}=1}=\frac{\lambda p}{2} v_{2} \bar{\lambda} K
\end{aligned}
$$

Let $L_{1}$ be the mean original buffer size. Since no customers exist in the original buffer when the server is idle and on vacation,

$$
L_{1}=L_{1,1} \text {. }
$$

Furthermore, let $L_{2}$ be the mean number of customers in the orbit:

$$
L_{2}=L_{2,0}+L_{2,1}+L_{2,2} \text {. }
$$

The mean total customers in the system including the one in service if any, denoted as $L_{s}$, can be obtained by using

$$
L_{s}=L_{1}+L_{2}+P_{B}
$$

3.3. Busy Cycle Analysis. Let $\Theta$ be the time between two consecutive vacations, which is called a busy cycle, $T_{V}, T_{I}$, and $T_{B}$, respectively, be the time periods during which the server is on vacation, idle, and busy, then $\Theta=$ $T_{V}+T_{I}+T_{B}$. Using the alternating renewal theory, we have that

$$
\frac{E\left[T_{V}\right]}{E[\Theta]}=\frac{\nu_{1}}{E[\Theta]}=P_{V}=\nu_{1} \bar{\lambda} K,
$$

which leads to

$$
E[\Theta]=\frac{1}{\bar{\lambda} K} .
$$

Furthermore, we obtain that

$$
\begin{aligned}
& E\left[T_{I}\right]=E[\Theta] P_{I}=\frac{\bar{\lambda}}{\lambda} \frac{G_{V}(\overline{\lambda p})}{\overline{\lambda p}}+\frac{1-G_{R}(\bar{\lambda})}{G_{R}(\bar{\lambda})} p\left(\nu_{1}-1\right), \\
& E\left[T_{B}\right]=E[\Theta] P_{B}=\frac{\mu_{1}}{1-\rho}\left(\lambda+\bar{\lambda} \frac{G_{V}(\overline{\lambda p})}{\overline{\lambda p}}+\frac{\lambda p}{G_{R}(\bar{\lambda})}\left(\nu_{1}-1\right)\right) .
\end{aligned}
$$

\section{Sojourn Time Distribution}

In this section, we consider the distribution of the sojourn time in the system of a test customer (TC). Let $T$ be the TC's sojourn time with p.g.f. $G_{T}(z)=\sum_{k=0}^{\infty} z^{k} P(T=k)$, which begins from the TC's arrival epoch to the TC's departure epoch.

To derive the expression of $G_{T}(z)$, let $D$ be the busy period in the standard Geo/G/1 queue, which begins at the epoch when one customer arrives and finds the server idle to begin his service immediately and ends at the epoch when one service is completed and the server becomes idle again. From Tian et al. [30], we know that the p.g.f. $G_{D}(z)$ of $D$ satisfies $G_{D}(z)=G_{B}\left(z\left(\bar{\lambda}+\lambda G_{D}(z)\right)\right)$ and $E[D]=$ $\mu_{1} / 1-\rho$.

Theorem 2. If $\rho<1$, the expression of the p.g.f. $G_{T}(z)$ can be given by

$$
\begin{aligned}
G_{T}(z)= & \bar{p}\left(P_{V}-\Pi_{1,2}(1)\right)+G_{B}(z)\left[P_{I}+\Pi_{1,2}(1)\right. \\
& +\left(\lambda+\frac{\bar{\lambda} G_{V}(\overline{\lambda p})}{\overline{\lambda p}}+\frac{\lambda p}{G_{R}(\bar{\lambda})}\left(\nu_{1}-1\right)\right) \omega(z) \\
& \left.+p\left(\bar{\lambda}+\lambda G_{D}(z)\right) \xi(z)(\psi(z)-\phi(z))\right] \bar{\lambda} K,
\end{aligned}
$$

where

$$
\begin{aligned}
\omega(z) & =\frac{G_{B}(z)-1}{z-\left(\bar{\lambda}+\lambda G_{B}(z)\right)} \\
\xi(z) & =\frac{G_{R}(\bar{\lambda} z)}{1-(\lambda / 1-\bar{\lambda} z)\left(1-G_{R}(\bar{\lambda} z)\right) G_{D}(z)}, \\
\tau(z) & =\xi(z) G_{D}(z), \\
\psi(z) & =\frac{G_{V}(z)-G_{V}(\overline{\lambda p}+\lambda p \tau(z))}{z-(\overline{\lambda p}+\lambda p \tau(z))} \\
\phi(z) & =\frac{G_{V}(\overline{\lambda p}+\lambda p \tau(z))}{\overline{\lambda p}+\lambda p \tau(z)} .
\end{aligned}
$$

Proof. Assume that the TC arrives in $\left(t, t^{+}\right)$, according to the state of the system at time $(t-1)^{-}$, we have that 


$$
\begin{aligned}
G_{T}(z)= & \lim _{t \rightarrow \infty} P\left(N_{2}\left((t-1)^{+}\right)=0, J\left((t-1)^{+}\right)=0\right) G_{B}(z) \\
& +\sum_{n_{2}=0}^{\infty} \sum_{i=1}^{\infty} \lim _{t \rightarrow \infty} P\left(N_{2}\left((t-1)^{+}\right)=n_{2}, U\left((t-1)^{+}\right)=i, J\left((t-1)^{+}\right)=0\right) G_{B}(z) \\
& +\sum_{n_{2}=0}^{\infty} \lim _{t \rightarrow \infty} P\left(N_{2}\left((t-1)^{+}\right)=n_{2}, U\left((t-1)^{+}\right)=1, J\left((t-1)^{+}\right)=2\right) G_{B}(z) \\
& +\sum_{n_{1}=0}^{\infty} \sum_{n_{2}=0}^{\infty} \sum_{i=1}^{\infty} \lim _{t \longrightarrow \infty} P\left(N_{1}\left((t-1)^{+}\right)=n_{1}, N_{2}\left((t-1)^{+}\right)=n_{2}, U\left((t-1)^{+}\right)=i, J\left((t-1)^{+}\right)=1\right) z^{i-1}\left(G_{B}(z)\right)^{n_{1}+1} \\
& +\bar{p} \lim _{t \longrightarrow \infty} \sum_{n_{2}=0}^{\infty} \sum_{i=2}^{\infty} P\left(N_{2}\left((t-1)^{+}\right)=n_{2}, U\left((t-1)^{+}\right)=i, J\left((t-1)^{+}\right)=2\right) \\
& +p \sum_{n_{2}=0}^{\infty} \sum_{i=2}^{\infty} \lim _{t \longrightarrow \infty} P\left(N_{2}\left((t-1)^{+}\right)=n_{2}, U\left((t-1)^{+}\right)=i, J\left((t-1)^{+}\right)=2\right) z^{i-1} T_{n_{2}}(z) \\
= & G_{B}(z)\left(P_{I}+\Pi_{1,2}(1)\right)+\frac{G_{B}(z)}{z} \Pi_{1}\left(z, G_{B}(z), 1\right)+\bar{P}\left(P_{V}-\Pi_{1,2}(1)\right)+\frac{p}{z} \sum_{n_{2}=0}^{\infty} \sum_{i=2}^{\infty} \pi_{n_{2}, i, 2} z^{i-1} E\left[z^{T_{n_{2}}}\right],
\end{aligned}
$$

where $T_{n_{2}}$ (with p.g.f $T_{n_{2}}(z)=E\left[z^{T_{n_{2}}}\right]$ ) denotes the sojourn time of the TC who is in the $\left(n_{2}+1\right)$-th position in the orbit, which begins at the epoch when the server completes his vacation and ends at the epoch when the TC leaves the system.

In the following, we finds the expression of $T_{n_{2}}(z)$. Assume that the vacation ends at the epoch $n$, considering whether an external customer arrives or not, we have the following.

Case 1. If there is an external arrival in the time interval $\left(n, n^{+}\right)$(with probability $\lambda$ ), the server will begin a busy period with length $D$, and then the customer at the head of the orbit can immediately begin retrying to access to the server. Let $\widehat{T}_{n_{2}}$ (with p.g.f. $\widehat{T}_{n_{2}}(z)$ ) be the sojourn time of the TC who is in the $\left(n_{2}+1\right)$-th position calculating from the epoch when the customer at the head of the orbit begins retrying to access to the server to the epoch when the TC leaves the system. In this case, we have $T_{n_{2}}=D+\widehat{T}_{n_{2}}$.

Case 2. If no external customer arrives in the time interval $\left(n, n^{+}\right)$(with probability $\bar{\lambda}$ ), then the customer at the head of the orbit immediately begins retrying to access to the server. In this case, we have $T_{n_{2}}=\widehat{T}_{n_{2}}$.

Then,

$$
T_{n_{2}}(z)=(\bar{\lambda}+\lambda D(z)) \widehat{T}_{n_{2}}(z) .
$$

However, for $\widehat{T}_{0}$, in this case, the TC is in the first position of the orbit, there exists two cases: one is that if the next external customer arrives later than the TC does, then $\widehat{T}_{0}$ equals the sum of the retrial time and the service time of the TC, i.e., $\widehat{T}_{0}=R+B$; the other is that if the next external customer arrives earlier than the TC does, then after a busy period with length $D$, the TC begins accessing to the server again; thus, $\widehat{T}_{0}=A+D+\widetilde{T}_{0}$, where $\widetilde{T}_{0}$ has the same distribution as $\widehat{T}_{0}$ and $A, D$, and $\widetilde{T}_{0}$ are independent random variables. Thus, we obtain that

$$
\begin{aligned}
\widehat{T}_{0}(z) & =\sum_{k=0}^{\infty} r_{k} \bar{\lambda}^{k} z^{k} G_{B}(z)+\sum_{k=1}^{\infty} \bar{\lambda}^{k-1} \lambda \sum_{m=k}^{\infty} r_{m} z^{k} G_{D}(z) \widehat{T}_{0}(z) \\
& =G_{R}(\bar{\lambda} z) G_{B}(z)+\frac{\lambda z}{1-\bar{\lambda} z}\left(1-G_{R}(\bar{\lambda} z)\right) G_{D}(z),
\end{aligned}
$$

which leads to

$$
\widehat{T}_{0}(z)=\frac{G_{R}(\bar{\lambda} z) G_{B}(z)}{1-(\lambda z / 1-\bar{\lambda} z)\left(1-G_{R}(\bar{\lambda} z)\right) G_{D}(z) T_{0}(z)} .
$$

Similarly, for $\widehat{T}_{n_{2}}, n_{2} \geq 1$, conditioning on whether the next external customer arrives earlier or later than the customer at the head of the orbit, we have

$$
\begin{aligned}
\widehat{T}_{n_{2}}(z)= & \sum_{k=0}^{\infty} r_{k} \bar{\lambda}^{k} z^{k} G_{D}(z) \widehat{T}_{n_{2}-1}(z) \\
& +\sum_{k=1}^{\infty} \bar{\lambda}^{k-1} \lambda \sum_{m=k}^{\infty} r_{m} z^{k} G_{D}(z) \widehat{T}_{n_{2}}(z) \\
= & G_{R}(\bar{\lambda} z) G_{D}(z) \widehat{T}_{n_{2}-1}(z)+\frac{\lambda}{1-\bar{\lambda} z} \\
& \cdot\left(1-G_{R}(\bar{\lambda} z)\right) G_{D}(z) \widehat{T}_{n_{2}}(z)
\end{aligned}
$$


which yields that

$$
\widehat{T}_{n_{2}}(z)=\tau(z) \widehat{T}_{n_{2}-1}(z) .
$$

It follows from (41)-(45), and we can obtain

$$
\begin{aligned}
& \widehat{T}_{n_{2}}(z)=(\tau(z))^{n_{2}+1} \frac{G_{B}(z)}{G_{D}(z)}, \\
& T_{n_{2}}(z)=\left(\bar{\lambda}+\lambda G_{D}(z)\right)(\tau(z))^{n_{2}+1} \frac{G_{B}(z)}{G_{D}(z)} .
\end{aligned}
$$

Substituting (46) into (40) leads to

$$
\begin{aligned}
G_{T}(z)= & \bar{p}\left(P_{V}-\Pi_{1,2}(1)\right)+G_{B}(z)\left[P_{I}+\Pi_{1,2}(1)\right. \\
& +\frac{\Pi_{1}\left(z, G_{B}(z), 1\right)}{z}+p\left(\bar{\lambda}+\lambda G_{D}(z)\right) \xi(z) \\
& \left.\cdot\left(\frac{\Pi_{2}(z, \tau(z))}{z}-\Pi_{1,2}(\tau(z))\right)\right] .
\end{aligned}
$$

By equation (13) and Theorem 1, the above equation can be changed as

$$
\begin{aligned}
G_{T}(z)= & \bar{p}\left(P_{V}-\Pi_{1,2}(1)\right)+G_{B}(z)\left[P_{I}+\Pi_{1,2}(1)\right. \\
& +\left(\lambda+\frac{\bar{\lambda} G_{V}(\overline{\lambda p})}{\overline{\lambda p}}+\frac{\lambda p}{G_{R}(\bar{\lambda})}\left(v_{1}-1\right)\right) \omega(z) \\
& \left.+p\left(\bar{\lambda}+\lambda G_{D}(z)\right) \xi(z)(\psi(z)-\phi(z))\right] \bar{\lambda} K,
\end{aligned}
$$

which completes the proof of Theorem 2 .

Using Theorem 2 and $G_{T}(1)=G_{D}(1)=\xi(1)=\tau(1)$ $=1, \omega(1)=\left(\mu_{1} / 1-\rho\right), \psi(1)-\phi(1)=\nu_{1}-1$, we can obtain the mean sojourn time in the system of an arbitrary TC as follows:

$$
\begin{aligned}
E(T)= & \left.\frac{\mathrm{d}}{\mathrm{d} z} G_{T}(z)\right|_{z=1} \\
= & \left.\left(1-\bar{p} P_{\mathrm{v}}+\bar{p} \Pi_{1,2}(1)\right) \frac{\mathrm{d}}{\mathrm{d} z} G_{B}(z)\right|_{z=1} \\
& +\bar{\lambda} K\left[\left.\left(\lambda+\frac{\bar{\lambda} G_{V}(\overline{\lambda p})}{\overline{\lambda p}}+\frac{\lambda p}{G_{R}(\bar{\lambda})}\left(\nu_{1}-1\right)\right) \frac{\mathrm{d}}{\mathrm{d} z} \omega(z)\right|_{z=1}\right. \\
& +p\left(\left.\lambda \frac{\mathrm{d}}{\mathrm{d} z} G_{D}(z)\right|_{z=1}+\left.\frac{\mathrm{d}}{\mathrm{d} z} \xi(z)\right|_{z=1}\right)(\psi(1)-\phi(1)) \\
& \left.+p\left(\left.\frac{\mathrm{d}}{\mathrm{d} z} \psi(z)\right|_{z=1}-\left.\frac{\mathrm{d}}{\mathrm{d} z} \phi(z)\right|_{z=1}\right)\right]
\end{aligned}
$$

After some laborious calculations, we have

$$
\begin{aligned}
\left.\frac{\mathrm{d}}{\mathrm{d} z} \omega(z)\right|_{z=1} & =\frac{\mu_{2}}{2(1-\rho)^{2}}, \\
\left.\frac{\mathrm{d}}{\mathrm{d} z} \xi(z)\right|_{z=1} & =\left(\frac{1}{\lambda}+\frac{\mu_{1}}{1-\rho}\right)\left(\frac{1}{G_{R}(\bar{\lambda})}-1\right), \\
\left.\frac{\mathrm{d}}{\mathrm{d} z} \tau(z)\right|_{z=1} & =\left(\frac{1}{\lambda}+\frac{\mu_{1}}{1-\rho}\right) \frac{1}{G_{R}(\bar{\lambda})}-\frac{1}{\lambda}, \\
\left.\frac{\mathrm{d}}{\mathrm{d} z} \psi(z)\right|_{z=1} & =\frac{\nu_{2}}{2}\left(\bar{p}+p\left(\frac{1}{(1-\rho) G_{R}(\bar{\lambda})}-1\right)\right), \\
\left.\frac{\mathrm{d}}{\mathrm{d} z} \phi(z)\right|_{z=1} & =p\left(\nu_{1}-1\right)\left(\frac{1}{(1-\rho) G_{R}(\bar{\lambda})}-1\right) .
\end{aligned}
$$

Inserting the above equations into (49) leads to

$$
\begin{aligned}
E(T)= & \mu_{1}\left(1-\bar{p} P_{\mathrm{v}}+\bar{p} \Pi_{1,2}(1)\right)+\bar{\lambda} K\left[\frac{\mu_{2}}{2(1-\rho)^{2}}\right. \\
& \cdot\left(\lambda+\frac{\bar{\lambda} G_{V}(\overline{\lambda p})}{\overline{\lambda p}}+\frac{\lambda p}{G_{R}(\bar{\lambda})}\left(\nu_{1}-1\right)\right) \\
& +p \frac{\nu_{2}}{2}\left(\bar{p}+\frac{p}{(1-\rho) G_{R}(\bar{\lambda})}\right) \\
& +p\left(\nu_{1}-1\right)\left(\frac{\rho}{1-\rho}+\left(\frac{1}{\lambda}+\frac{\mu_{1}}{1-\rho}\right)\left(\frac{1}{G_{R}(\bar{\lambda})}-1\right)\right. \\
& \left.\left.-p\left(\frac{1}{(1-\rho) G_{R}(\bar{\lambda})}-1\right)\right)\right] .
\end{aligned}
$$

Here, we can verify that

$$
\begin{aligned}
\frac{P_{B}}{\lambda}= & \frac{\mu_{1}}{1-\rho}\left(1+\frac{\bar{\lambda}}{\lambda} \frac{G_{V}(\overline{\lambda p})}{\overline{\lambda p}}\right. \\
& \left.+\frac{p}{G_{R}(\bar{\lambda})}\left(\nu_{1}-1\right)\right) \bar{\lambda} K \\
= & \mu_{1}\left(1-\bar{p} P_{\mathrm{v}}+\bar{p} \Pi_{1,2}(1)\right) \\
\frac{L_{1,1}}{\lambda}= & \frac{\mu_{2}}{2(1-\rho)^{2}} \\
& +\left(\lambda+\frac{\bar{\lambda} G_{V}(\overline{\lambda p})}{\overline{\lambda p}}+\frac{\lambda p}{G_{R}(\bar{\lambda})}\left(\nu_{1}-1\right)\right) \bar{\lambda} K, \\
\frac{L_{2,0}+L_{2,1}+L_{2,2}}{\lambda=} & p\left[( \nu _ { 1 } - 1 ) \left(\frac{\rho}{1-\rho}\right.\right. \\
& +\left(\frac{1}{\lambda}+\frac{\mu_{1}}{1-\rho}\right)\left(\frac{1}{G_{R}(\bar{\lambda})}-1\right) \\
& \left.-p\left(\frac{1}{(1-\rho) G_{R}(\bar{\lambda})}-1\right)\right) \\
& \left.+\frac{\nu_{2}}{2}\left(\bar{p}+\frac{p}{(1-\rho) G_{R}(\bar{\lambda})}\right)\right] \bar{\lambda} K .
\end{aligned}
$$


Therefore, we obtain that

$$
E(T)=\frac{P_{B}+L_{1,1}+L_{2,0}+L_{2,1}+L_{2,2}}{\lambda}=\frac{L_{S}}{\lambda},
$$

which shows that Little's law holds for our discrete-time Geo/ G/1 retrial queue with sever vacation and two waiting buffers.

\section{Sensitivity Analysis and Cost Optimization}

In this section, we present some numerical examples to show the effect of some parameters on performance measures of the system (i.e., sensitivity analysis) and the optimization problem based on a given cost structure. We assume that the arrival rate $\lambda=0.45$, service time follows a discrete phasetype distribution $\mathrm{PH}_{d}(\alpha, T), \boldsymbol{\alpha}=(1 / 3,2 / 3), \mathbf{T}=\left(\begin{array}{cc}1 / 2 & 0 \\ 0 & 1 / 3\end{array}\right)$, $\mu_{1}=5 / 3$, and $\rho=0.75$. The retrial time $R$ follows a discrete phase-type distribution $\mathrm{PH}_{d}(\beta, S), \boldsymbol{\beta}=(1 / 5,4 / 5)$, and $\mathbf{S}=$ $\left(\begin{array}{cc}1 / 2 & 0 \\ 0 & 3 / 4\end{array}\right)$

In the following subsection, we first give the sensitivity analysis based on the above results.

5.1. Sensitivity Analysis. Considering different distributions of vacation time $V$, respectively, with deterministic distribution with p.g.f $G_{V}(z)=z^{D}$ and negative binomial distribution with p.g.f $G_{V}(z)=(v z /(1-\bar{v} z))^{2}$, we present the effect of $D$ and $v$ on $P_{B}, E[T]$, and $E[\Theta]$ for different values of $p$, respectively, in Figures 2-4 and in Figures 5-7. Some findings are given as follows.

(1) If $p=1, P_{B}$ takes the fixed value no matter what distribution the vacation time follows, which is because when $p=1$, all customers who arrive at the system during the vacation period enter the orbit and no customer is lost, and the busy probability $P_{B}$ is the same as that in the standard $\mathrm{Geo} / \mathrm{G} / 1$ queue, i.e., $P_{B}=\rho$.

(2) Figure 2 shows that when $D=1$, the value of $p$ has no effect on $P_{B}, E[T]$, and $E[\Theta]$, i.e., $P_{B}, E[T]$, and $E[\Theta]$, respectively, take fixed values, which are corresponding to the busy probability, mean sojourn time, and mean busy cycle in the standard Geo/G/1 queue.

(3) $P_{B}, E[T]$, and $E[\Theta]$ are increasing as $p$ increases given that vacation time (i.e., $D$ or $v$ ) is fixed and is larger than 1 . The reason is that as $p$ increases, more customers who arrive during the vacation period enter the orbit, which leads to the increase in busy period and thus makes the server's busy probability, mean sojourn time of the TC, and mean busy cycle increase.

(4) Under given $p, P_{B}$ is decreasing as mean vacation time increases, but $E[T]$ and $E[\Theta]$ are increasing as mean vacation time increases, which agrees with our expectation.

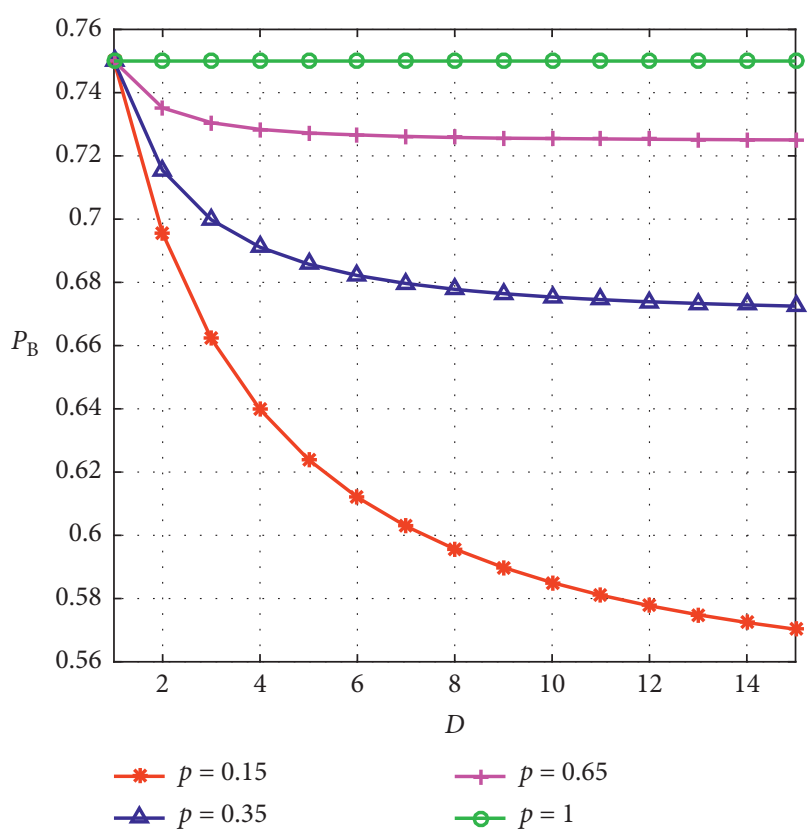

Figure 2: Effect of $D$ on $P_{B}$ for different $p$ values.

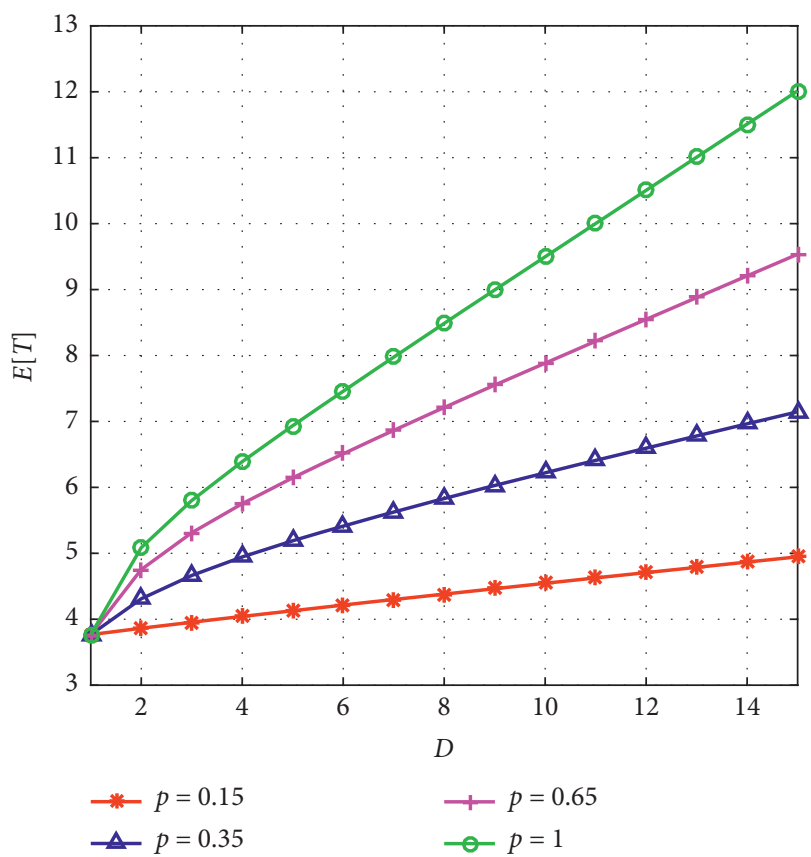

Figure 3: Effect of $D$ on $E[T]$ for different $p$ values.

5.2. Cost Model. In the sequel, we will discuss the optimal vacation length under a given cost assumption because determining an optimal policy to achieve the minimum cost is very important in queueing theory. For the discretetime Geo/G/1 retrial queue with two waiting buffers and a fixed vacation time, we consider the cost elements as follows:

$C_{\mathrm{h} 1} \equiv$ unit time cost of every customer in the original buffer 


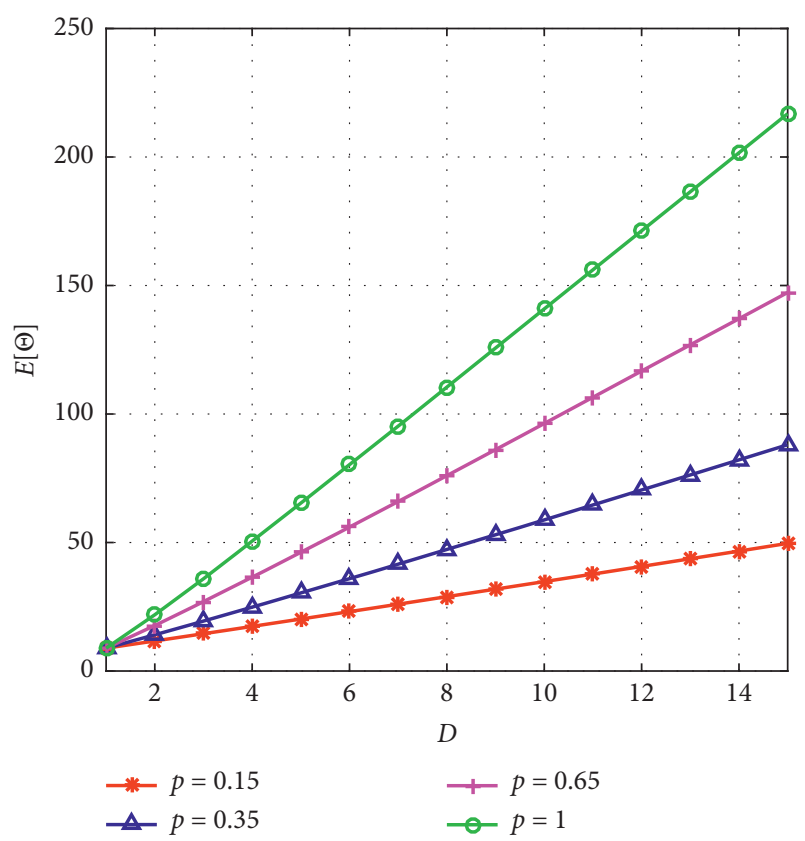

FIgURE 4: Effect of $D$ on $E[\Theta]$ for different $p$ values.

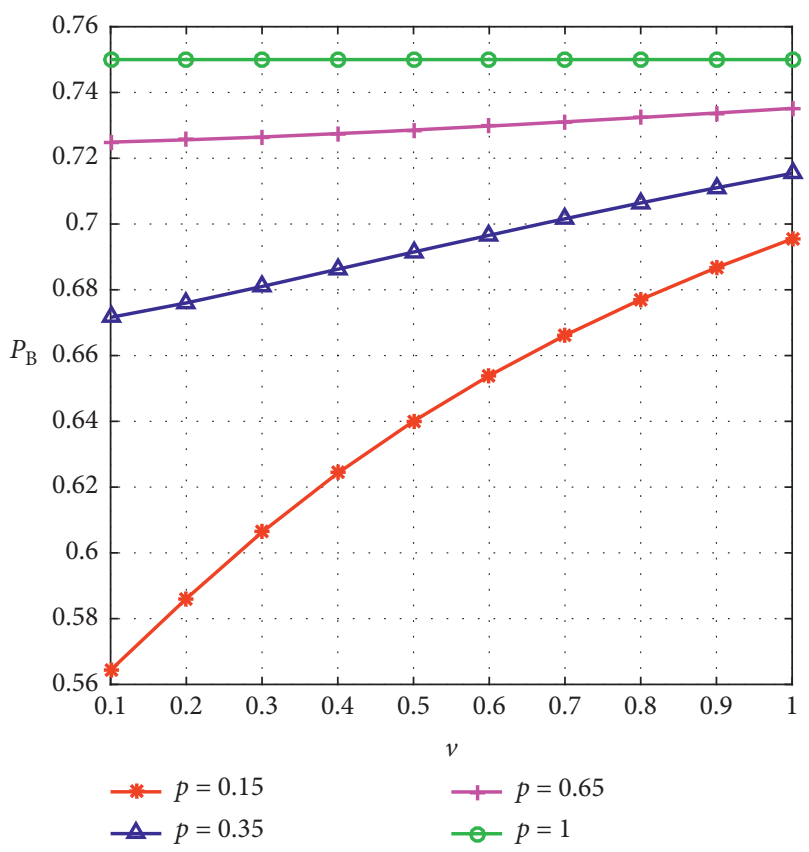

Figure 5: Effect of $v$ on $P_{B}$ for different $p$ values.

$C_{\mathrm{h} 2} \equiv$ unit time cost of every customer in the orbit

$C_{0} \equiv$ unit time cost for keeping the server idle

$C_{1} \equiv$ unit time cost for keeping the server busy

$C_{2} \equiv$ unit time cost for the server being on vacation

$C_{\mathrm{s}} \equiv$ setup cost per cycle

Firstly, assuming that the vacation time $V$ follows deterministic distribution with fixed length $D, D \geq 1$ rather than the variable, then $v_{1}=D$ and $v_{2}=D(D-1)$. Taking $D$ as the decision variable, our objective is to find the optimal

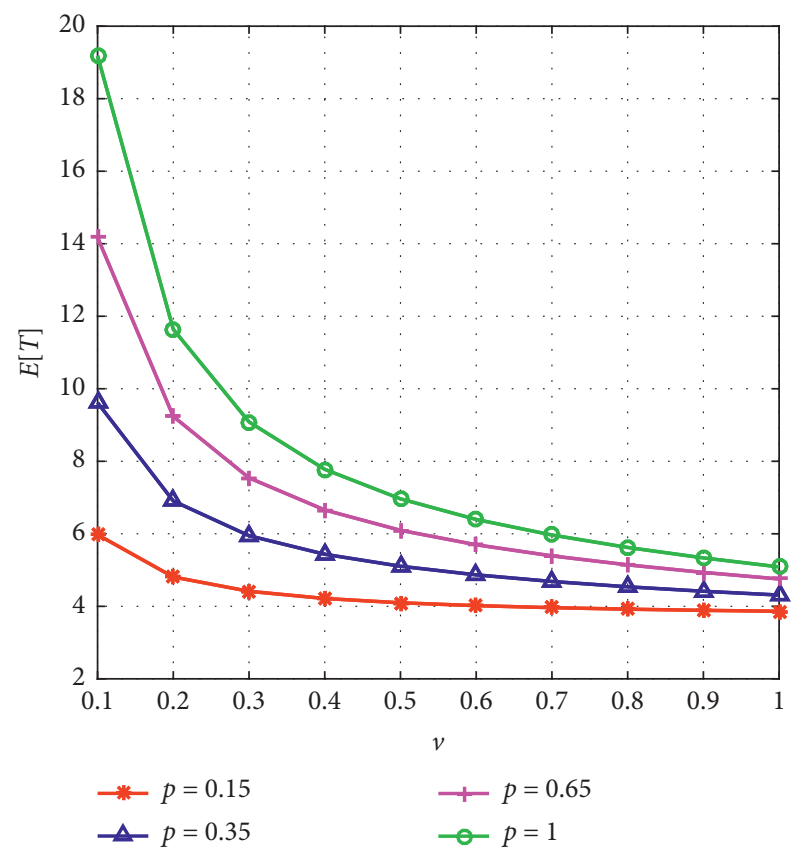

FIgURE 6: Effect of $v$ on $E[T]$ for different $p$ values.

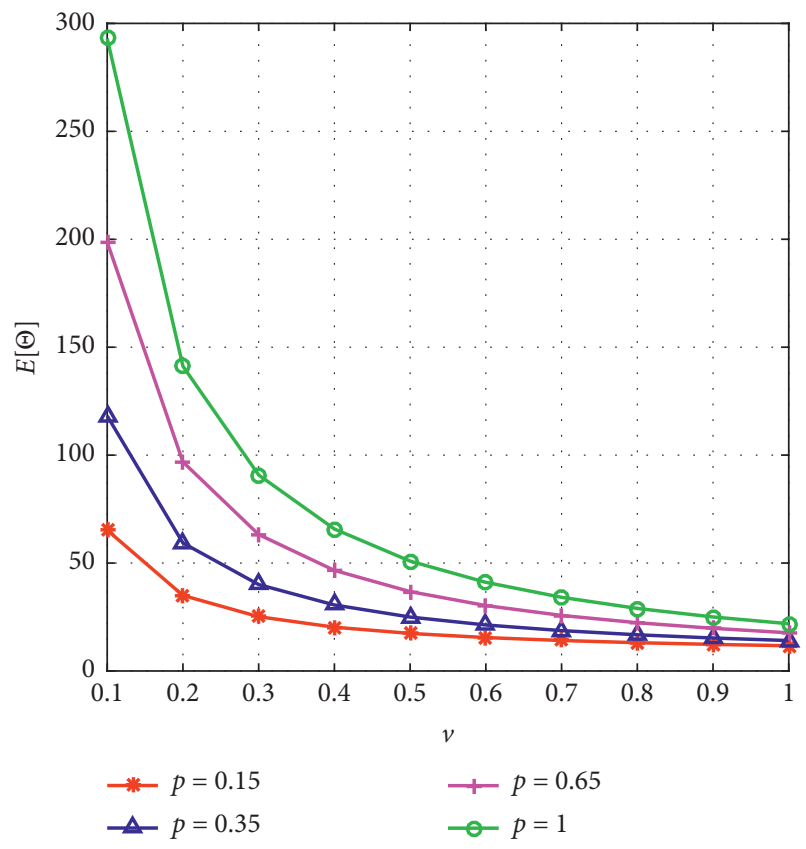

Figure 7: Effect of $v$ on $E[\Theta]$ for different $p$ values.

value $D$, say $D^{*}$ to minimize the cost function $C(D)$, where $C(D)$ denotes the long-run average cost per unit time and can be expressed as

$$
C(D)=C_{h 1} L_{1}+C_{h 2} L_{2}+C_{0} P_{I}+C_{1} P_{B}+C_{2} P_{V}+C_{s} \frac{1}{E[\Theta]} .
$$

The cost minimization problem can be mathematically illustrated as follows: 


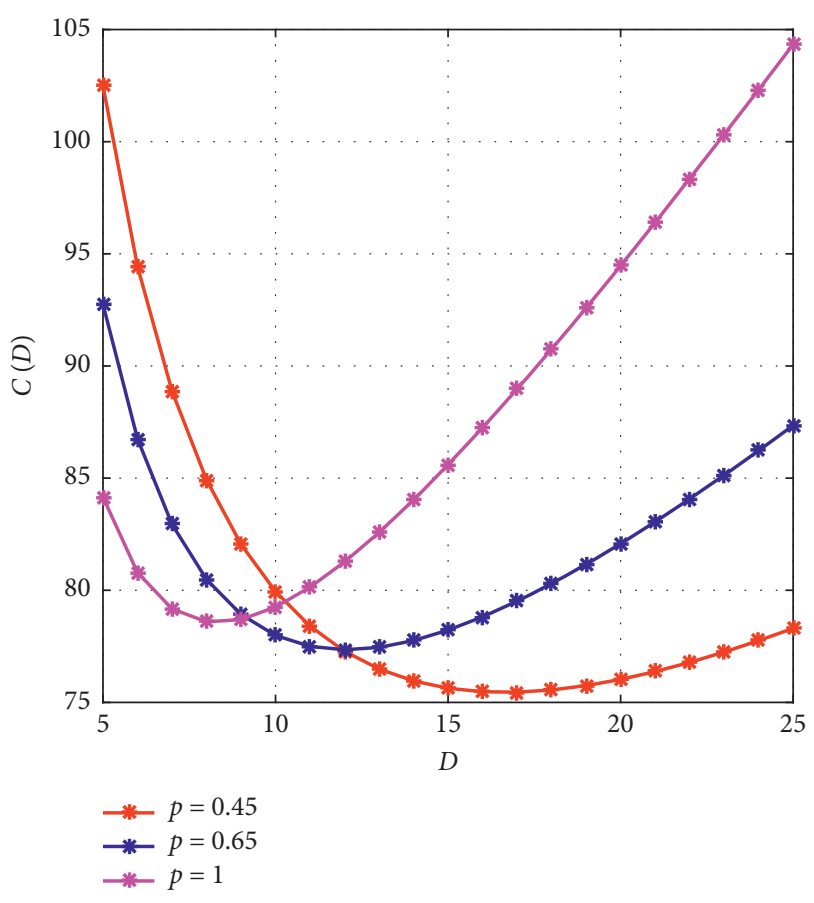

Figure 8: $C(D)$ v.s. $D$ for $p=0.45,0.65$, and 1 .

$$
C\left(D^{*}\right)=\min _{D} C(D),
$$

subject to $\quad D \geq 1$.

Owing to the highly nonlinear and complex nature of the optimization problem, it is an arduous task to find the optimal solution $D^{*}$ analytically. In the following, we present some numerical examples for illustrative purposes and find the optimum solution $D^{*}$ by Matlab. Taking $C_{s}=$ 2000, $C_{h 1}=20, C_{h 2}=10, C_{0}=7, C_{1}=25$, and $C_{2}=25$, we depict the effect of $D$ on the cost function $C(D)$ for different values of $p=0.45,0.65$, and 1 in Figure 8 .

Similarly, in the second numerical example, we consider that the vacation time $V$ follows negative binomial distribution with p.g.f $G_{V}(z)=(v z / 1-\bar{v} z)^{2}$ and take $v$ as the decision variable, and then the cost minimization problem can be mathematically illustrated as follows:

$$
\begin{aligned}
& \qquad C\left(v^{*}\right)=\min _{v} C(v), \\
& \text { subject to } 0<v \leq 1,
\end{aligned}
$$

where

$$
C(v)=C_{h 1} L_{1}+C_{h 2} L_{2}+C_{0} P_{I}+C_{1} P_{B}+C_{2} P_{V}+C_{s} \frac{1}{E[\Theta]}
$$

We present the effect of $v$ on the cost function $C(v)$ for different values of $p=0.45,0.65$, and 1 in Figure 9, where $C_{s}=2000, C_{h 1}=20, C_{h 2}=10, C_{0}=7, C_{1}=25$, and $C_{2}=25$.

From Figures 8 and 9, we can see that the optimal values $D^{*}$ and $v^{*}$ and minimal values $C\left(D^{*}\right)$ and $C\left(v^{*}\right)$ exist and are given in Table 1 , which can be obtained by applying the

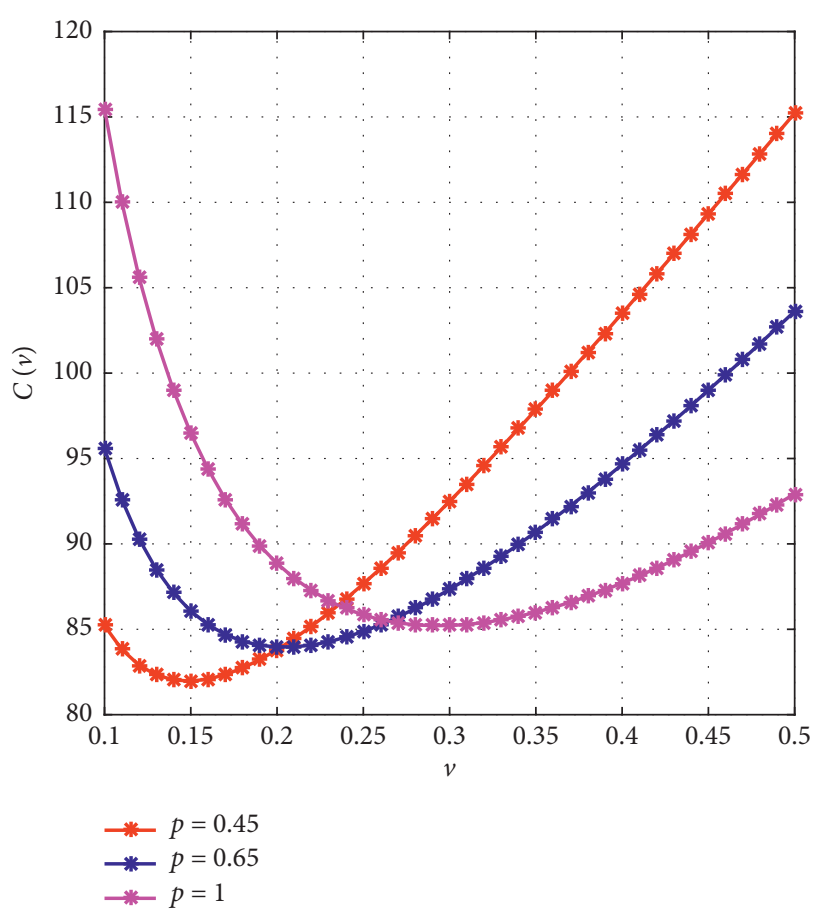

Figure 9: $C(v)$ v.s. $v$ for $p=0.45,0.65$, and 1 .

Table 1: Optimal values $D^{*}$ and $v^{*}$ and minimum values $C\left(D^{*}\right)$ and $C\left(v^{*}\right)$

\begin{tabular}{lcccc}
\hline & \multicolumn{2}{c}{$\begin{array}{c}\text { Deterministic } \\
\text { distribution }\end{array}$} & \multicolumn{2}{c}{$\begin{array}{c}\text { Negative binomial } \\
\text { distribution }\end{array}$} \\
& $D^{*}$ & $C\left(D^{*}\right)$ & $v^{*}$ & $C\left(v^{*}\right)$ \\
\hline$p=0.45$ & 17 & 75.4573 & 0.1484 & 81.9648 \\
$p=0.65$ & 12 & 77.3477 & 0.2041 & 83.9525 \\
$p=1$ & 8 & 78.5946 & 0.2935 & 85.2095 \\
\hline
\end{tabular}

computer software Matlab. Table 1 exhibits that (i) $C\left(D^{*}\right)$ and $C\left(v^{*}\right)$ increase with increasing $p$; (ii) as $p$ increases, the optimal vacation time length decreases; i.e., $D^{*}$ decreases and $v^{*}$ increases.

\section{Conclusions}

In this paper, based on the characteristics of the ATM network, we presented a discrete-time Geo/G/1 retrial queue with two waiting buffers and single vacation, where retrial customers are incurred by the server's single vacation; that is, arriving customers during the server vacation period either choose to join the retrial buffer or leave the system without service. We studied some important system characteristics by using the supplementary variable method and generating the function approach. Distribution of the sojourn time of any arbitrary customer was studied by using the generating function method. Finally, we presented the sensitivity and cost optimization analysis. As further potential future study, we can generalize this retrial queueing model with two waiting buffers to some different cases, as follows: 
(i) Retrial customers are incurred by the unavailability of the server, in which an arriving customer who find the server busy or on vacation either joins the waiting buffer with probability $p_{1}$ or joins the retrial buffer with probability $p_{2}$ or leaves the system for ever with probability $p_{3}$, where $p_{1}+p_{2}+p_{3}=1$.

(ii) Retrial queues with working vacation policy, where retrial customers are incurred by the lower service rate of the server during the working vacations.

(iii) In view of the feedback phenomenon in the retrial queue, one can consider the case that retrial customers are incurred by those feedback customers.

(iv) In reality, the capacity of the buffer in service systems is often finite. Hence, one can consider that an arriving customer who finds no vacant space either enters a retrial buffer or leaves the system for ever.

\section{Data Availability}

The data used to support the findings of this study are included within the article. The reader can directly access the data in the work.

\section{Conflicts of Interest}

The authors declare that there are no conflicts of interest regarding the publication of this paper.

\section{Acknowledgments}

This research was supported by the Natural Science Foundation of Anhui Higher Education Institutions of China (Grant nos. KJ2017A340 and KJ2019A0535), Fuyang Municipal Government-Fuyang Normal University Horizontal Cooperation projects in 2017 (no. XDHXTD201709), and the National Natural Science Foundation of China (no. 61672006).

\section{References}

[1] H. Bruneel and B. G. Kim, Discrete-Time Models for Communication Systems Including ATM, Kluwer Academic Publishers, Boston, MA, USA, 1993.

[2] H. Takagi, Queueing Analysis: Discrete-ime Systems, Northolland Elsevier, vol. 3, Amsterdam, Netherlands, 1993.

[3] V. Ramaswami and J. L. Wang, "A hybrid analysis/simulation for ATM performance with application to quality-of-service of CBR traffic," Telecommunication Systems, vol. 5, no. 1, pp. 25-48, 1996.

[4] J. Walraevens, B. Steyaert, and H. Bruneel, "Performance analysis of a single-server ATM queue with a priority scheduling," Computers \& Operations Research, vol. 30, no. 12, pp. 1807-1829, 2003.

[5] G. I. Falin and J. G. C. Templeton, Retrial Queues, Chapman \& Hall, London, UK, 1997.

[6] J. R. Artalejo and A. Gomez-Corral, Retrial Queueing Systems, Springer, Berlin, Germany, 2008.

[7] J. R. Artalejo, "A classified bibliography of research on retrial queues: progress in 1990-1999,” Top, vol. 7, no. 2, pp. 187-211, 1999.
[8] J. R. Artalejo, "Accessible bibliography on retrial queues: progress in 2000-2009," Mathematical and Computer Modelling, vol. 51, no. 9-10, pp. 1071-1081, 2010.

[9] A. Gómez-Corral, "A bibliographical guide to the analysis of retrial queues through matrix analytic techniques," Annals of Operations Research, vol. 141, no. 1, pp. 163-191, 2006.

[10] J. Kim and B. Kim, "A survey of retrial queueing systems," Annals of Operations Research, vol. 247, no. 1, pp. 3-36, 2016.

[11] S. Gao, J. Wang, and T. Van Do, "A repairable retrial queue under Bernoulli schedule and general retrial policy," Annals of Operations Research, vol. 247, no. 1, pp. 169-192, 2016.

[12] I. Dimitriou, "A two-class queueing system with constant retrial policy and general class dependent service times," European Journal of Operational Research, vol. 270, no. 3, pp. 1063-1073, 2018.

[13] M. Dai, J. Xia, H. Xia, and H. Shen, "Event-triggered passive synchronization for Markov jump neural networks subject to randomly occurring gain variations," Neurocomputing, vol. 331, pp. 403-411, 2019.

[14] H. Shen, M. Chen, Z.-G. Wu, J. Cao, and J. H. Park, "Reliable event-triggered asynchronous extended passive control for semi-markov jump fuzzy systems and its application," IEEE Transactions on Fuzzy Systems, 2019.

[15] H. Shen, T. Wang, J. Cao, G. Lu, Y. Song, and T. Huang, "Nonfragile dissipative synchronization for Markovian memristive neural networks: a gain-scheduled control scheme," IEEE Transactions on Neural Networks and Learning Systems, vol. 30, no. 6, pp. 1841-1853, 2019.

[16] Z. G. Zhang and N. Tian, "Discrete-time Geo/G/1 queue with multiple adaptive vacations," Queueing Systems, vol. 38, no. 4, pp. 419-429, 2001.

[17] S. Gao and J. Wang, "Discrete-Time Geo/G/1 retrial queue with general retrial times, working vacations and vacation interruption," Quality Technology \& Quantitative Management, vol. 10, no. 4, pp. 495-512, 2013.

[18] J. Wang, "Discrete-time Geo/G/1 retrial queues with general retrial time and Bernoulli vacation," Journal of Systems Science and Complexity, vol. 25, no. 3, pp. 504-513, 2012.

[19] T.-Y. Wang, J.-C. Ke, and F.-M. Chang, "On the discrete-time Geo/G/1 queue with randomized vacations and at most J vacations," Applied Mathematical Modelling, vol. 35, no. 5, pp. 2297-2308, 2011.

[20] D. Yue and F. Zhang, "A discrete-time Geo/G/1 retrial queue with J-vacation policy and general retrial times," Journal of Systems Science and Complexity, vol. 26, no. 4, pp. 556-571, 2013.

[21] C. Luo, Y. Tang, B. Chao, and K. Xiang, "Performance analysis of a discrete-time Geo / G / 1 queue with randomized vacations and at most J vacations," Applied Mathematical Modelling, vol. 37, no. 9, pp. 6489-6504, 2013.

[22] T.-Y. Wang, "An unreliable Geo/G/1 queue with startup and closedown times under randomized finite vacations," Applied Mathematical Modelling, vol. 39, no. 3-4, pp. 1383-1399, 2015.

[23] T.-Y. Wang, "A $\langle V, p\rangle$-policy queueing system with startup/ closedown times," Quality Technology \& Quantitative Management, vol. 12, no. 2, pp. 169-194, 2015.

[24] B. Krishna Kumar, R. Rukmani, and V. Thangaraj, "On multiserver feedback retrial queue with finite buffer," Applied Mathematical Modelling, vol. 33, no. 4, pp. 2062-2083, 2009.

[25] T. V. Do, "An efficient computation algorithm for a multiserver feedback retrial queue with a large queueing capacity," Applied Mathematical Modelling, vol. 34, no. 8, pp. 22722278, 2010. 
[26] G. I. Falin, "The $M / M / 1$ retrial queue with retrials due to server failures," Queueing Systems, vol. 58, no. 3, pp. 155-160, 2008.

[27] G. I. Falin, “An M/G/1 retrial queue with an unreliable server and general repair times," Performance Evaluation, vol. 67, no. 7, pp. 569-582, 2010.

[28] N. Sherman and J. Kharoufeh, "Optimal Bernoulli Routing in an unreliable M/G/1 retrial queue," Probability in the Engineering and Informational Sciences, vol. 25, no. 1, pp. 1-20, 2011.

[29] H. Li and T. Yang, "Geo/G/1 discrete time retrial queue with Bernoulli schedule," European Journal of Operational Research, vol. 111, no. 3, pp. 629-649, 1998.

[30] N. Tian, X. Xu, and Z. Ma, Discrete-Time Queueing Theory, Science press, Beijing, China, 2008. 


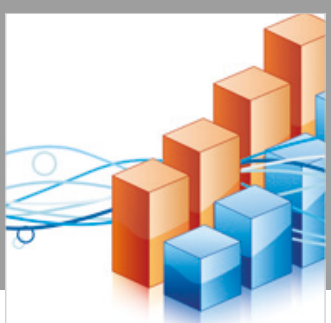

Advances in

Operations Research

\section{-n-m}
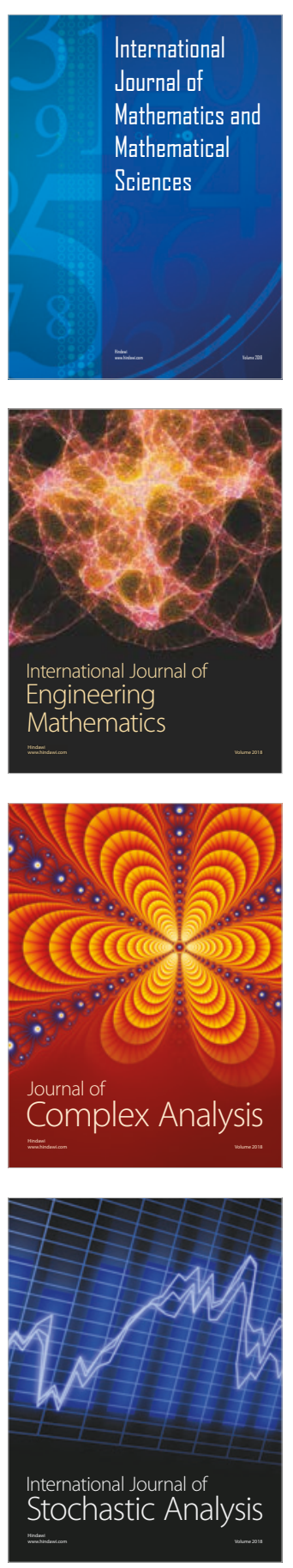
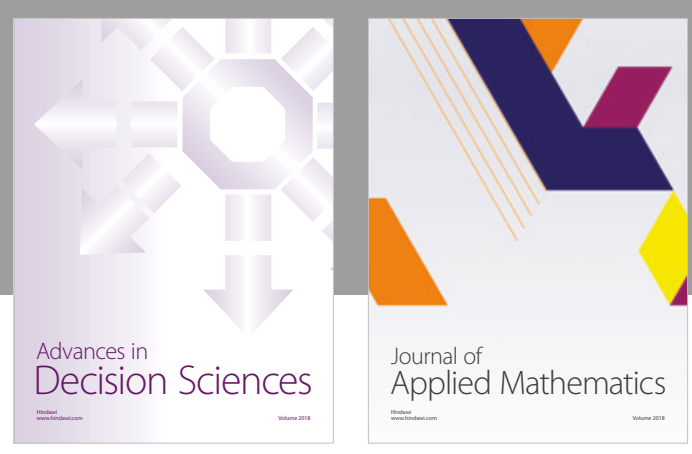

Journal of

Applied Mathematics
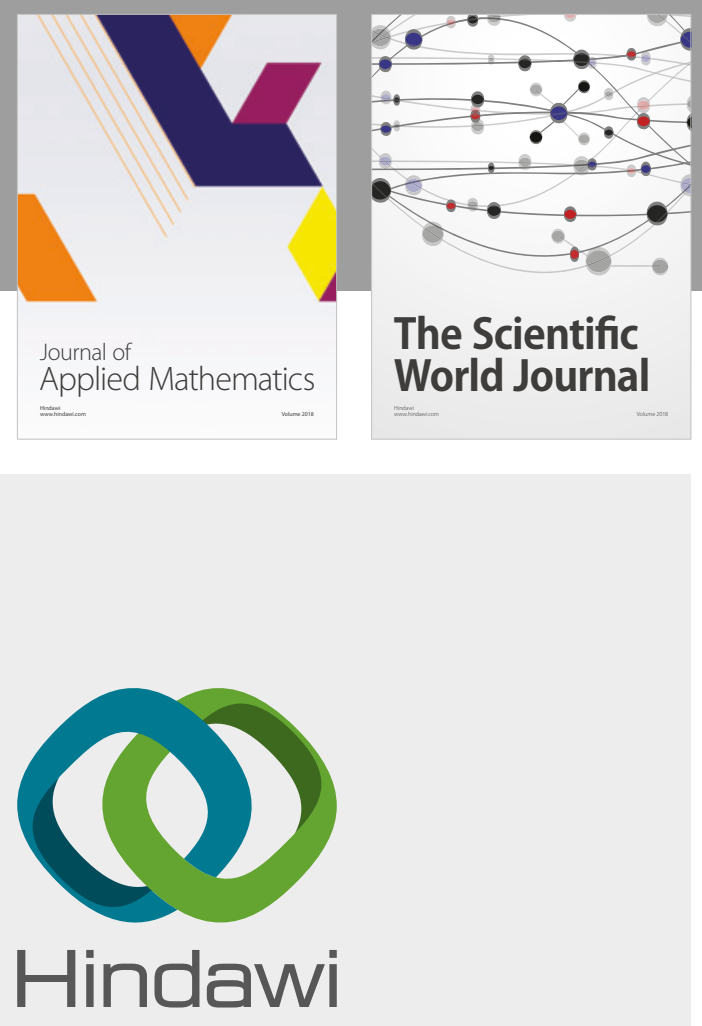

Submit your manuscripts at

www.hindawi.com

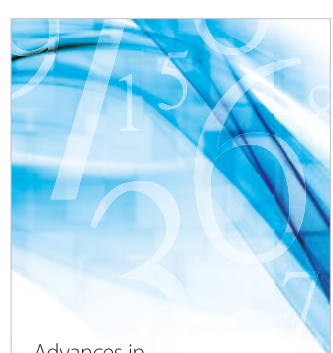

Advances in
Numerical Analysis
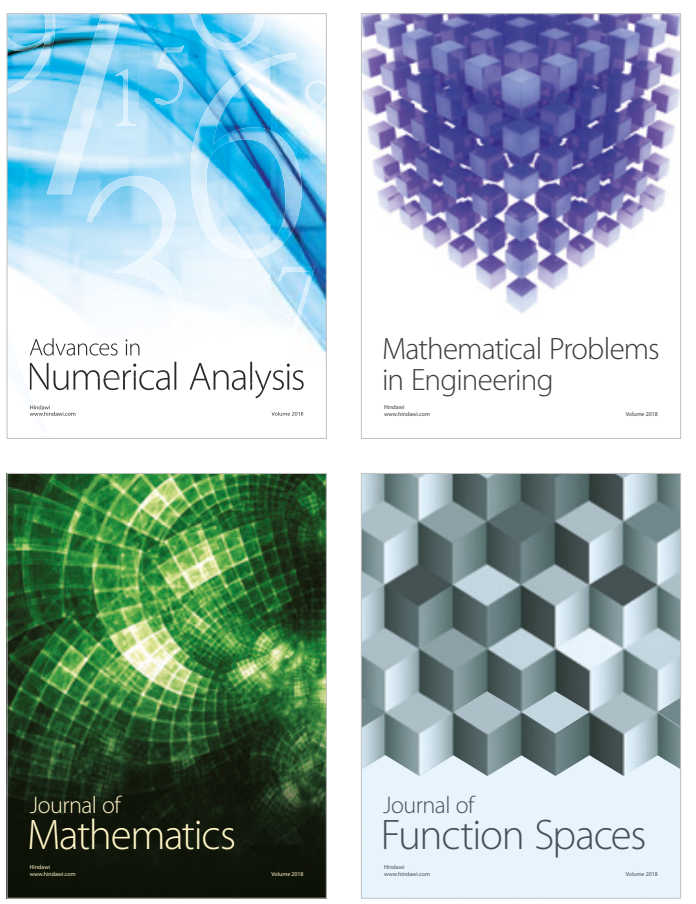

Mathematical Problems in Engineering

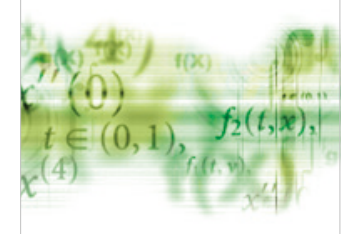

International Journal of

Differential Equations

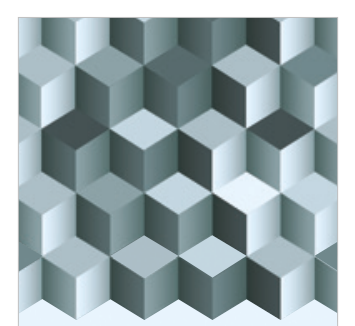

Journal of

Function Spaces

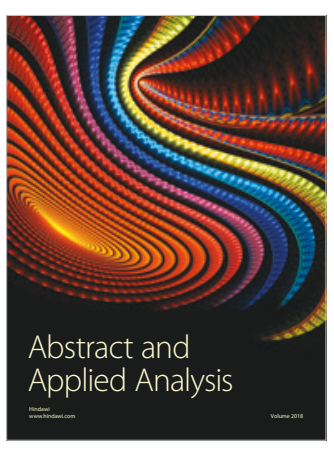

The Scientific

World Journal

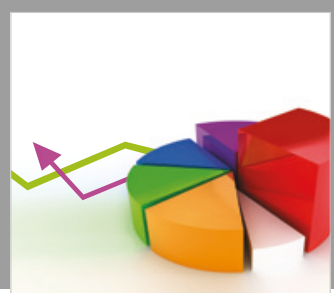

Journal of

Probability and Statistics
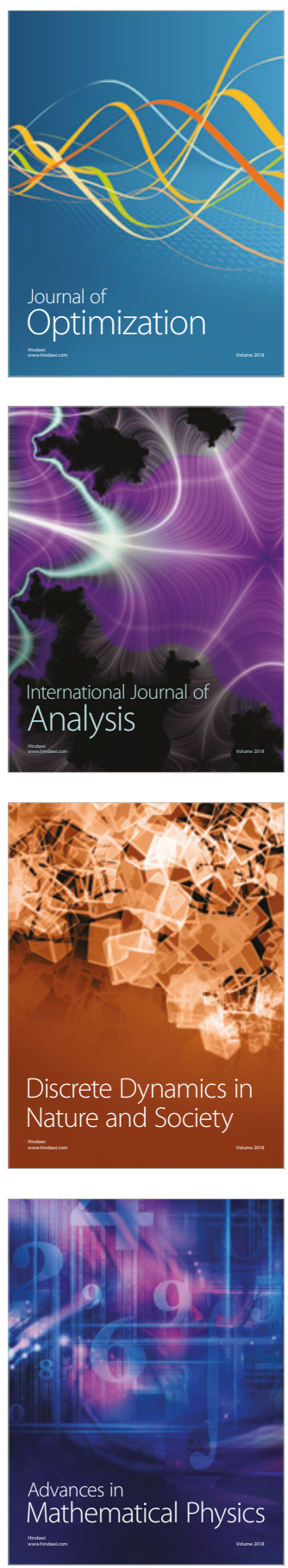